\title{
Elimination of inter- and intramolecular crosslinks of phosphorylated chitosan by sodium salt formation
}

\author{
Wanpen Tachaboonyakiat, Nitsana Netswasdi, Veeranuch Srakaew and Mantana Opaprakasit \\ Inter- and intramolecular crosslinking is the main cause of the water insolubility of phosphorylated chitosan synthesized with a \\ phosphorus pentoxide/methanesulfonic acid system. The goal of this study was to eliminate inter- and intramolecular crosslinks \\ and synthesize water-soluble phosphorylated chitosan. Two main factors for the formation of inter- and intramolecular crosslinks \\ are (i) electrostatic interaction between amino groups and methanesulfonic acid salt and (ii) electrostatic interaction between \\ ammonium groups and phosphoric groups. The influences of ethanol washing and sodium salt formation on the elimination of \\ inter- and intramolecular crosslinks were investigated. Sodium phosphorylated chitosan was water soluble. The solubility was \\ independent of the degree of phosphoric substitution.
}

Polymer Journal (2010) 42, 148-156; doi:10.1038/pj.2009.317; published online 23 December 2009

Keywords: crosslink; intermolecular; intramolecular; phophorylation; phosphorylated chitosan; sodium salt; water soluble

\section{INTRODUCTION}

Chitosan, (1-4)-2-amino-2-deoxy- $\beta$-D-glucopyranose, is a naturally occurring polysaccharide obtained industrially by the deacetylation of chitin. Chitin, (1,4)-2-acetamido-2-deoxy- $\beta$-D-glucopyranose, is extracted from the exoskeleton of crustaceans. Chitosan is nontoxic, biocompatible and biodegradable. It is soluble in most dilute acids but insoluble in water. Its poor water solubility has been a major drawback in its use in pharmaceutical and biomedical applications. Several attempts have been made to improve the water solubility of chitosan through several chemical modifications such as chitosan-graft-poly (ethylene glycol) methyl ether, ${ }^{1}$ chitosan-gallic acid ${ }^{2} N$ - $(\gamma$-propanoylamino acid)-chitosans, ${ }^{3} \mathrm{~N}$-acylated or $\mathrm{N}$-alkylated chitosan, ${ }^{4}$ $O$-succinyl-chitosan ${ }^{5}$ and chitosan-L-glutamic acid, ${ }^{6}$ among others. However, this research focuses on the chemical modification of chitosan with phosphoric groups. As phosphoric groups or phosphate groups are normally found in the cell membranes of any organism, chitosan modified with phosphoric groups could exhibit cytocompatibility, ${ }^{7,8}$ bioabsorbability ${ }^{7,8}$ and osteoinductiveness. ${ }^{7,8}$ Owing to the wide ranges of biomedical and pharmaceutical applications ${ }^{7-14}$ of phosphorylated chitosan, phosphorylation of chitin/chitosan has been reported through three main systems, namely (i) orthophosphoric acid/urea/dimethylformamide, ${ }^{15}$ (ii) $\mathrm{H}_{3} \mathrm{PO}_{4} / \mathrm{Et}_{3} \mathrm{PO}_{4} / \mathrm{P}_{2} \mathrm{O}_{5} /$ hexanol $^{16,17}$ or butanol ${ }^{18}$ and (iii) $\mathrm{P}_{2} \mathrm{O}_{5} /$ methanesulfonic acid. ${ }^{19,21}$ Other phosphate available chitin/chitosan derivatives have also been reviewed. ${ }^{22}$ Phosphorylation of chitin/chitosan with phosphorus pentoxide in methanesulfonic acid provides a high degree of substitution (DS). Methanesulfonic acid functions not only as a good solvent for chitin/ chitosan but also as an efficient catalyst for the esterification reaction. In the phosphorylation of chitin and deacetylated chitin, it was found that chitin phosphates are easily soluble in water independent of the DS, whereas deacetylated chitin phosphates with a high DS are water insoluble. This insoluble property is attributed to the formation of inter- or intramolecular linkage between amino groups-methanesulfonic acid salt and phosphoric groups. ${ }^{20,21}$ Normally, the insoluble product is ignored by filtering. To synthesize water-soluble chitosan derivatives with a high degree of phosphoric substitution, the elimination of inter- and intramolecular crosslinks by a simple method, referred to in this research as sodium salt formation, attracted our interest. The salt of alkaline metals is water soluble. Thus, to investigate the elimination of inter- and intramolecular crosslinks, the selection of washing solvents, acetone or ethanol, followed by sodium salt formation, was also examined. The structural characterization of water-soluble and water-insoluble products was determined by Fourier transform infrared spectrometer (FT-IR). The degree of phosphoric substitution was then analyzed by the percentage of the element ratio of phosphorus to nitrogen obtained using a scanning electron microscope (SEM) equipped with an energy-dispersive X-ray spectrometer. Solubility in water, acids and bases was determined to provide information for biomedical applications over a wide $\mathrm{pH}$ range.

\section{EXPERIMENTAL PROCEDURE}

Materials

Chitosan with a low viscosity (MW 371000 determined by high-performance liquid chromatography, $\mathrm{DD}=85$ determined by FT-IR) was purchased from Fluka (Tokyo, Japan). Methanesulfonic acid and phosphorus pentoxide were obtained from Acros Organics (Morris Plains, NJ, USA). Acetic acid was purchased from Labscan Asia Co. Ltd. (Bangkok, Thailand). Sodium hydrogen

Faculty of Science, Department of Materials Science, Chulalongkorn University, Phyathai, Patumwan, Bangkok, Thailand

Correspondence: Dr W Tachaboonyakiat, Faculty of Science, Department of Materials Science, Chulalongkorn University, Phyathai Road, Patumwan, Bangkok 10330, Thailand. E-mail: wanpen.ta@chula.ac.th

Received 9 July 2009; revised 13 October 2009; accepted 14 October 2009; published online 23 December 2009 
carbonate was bought from Merck (Darmstadt, Germany). Commercial grade acetone and ethanol were obtained from Zen Point (Bangkok, Thailand). All chemicals were used without further purification.

\section{Synthesis of phosphorylated chitosan}

The synthesis of phosphorylated chitosan was modified from the synthesis of phosphorylated chitin and deacetylated chitin as reported by Nishi et al ${ }^{19-21}$ Chitosan of $4 \mathrm{~g}$ was dissolved in $28 \mathrm{ml}$ of concentrated methanesulfonic acid using a mechanical stirrer at a speed of 500 r.p.m. Phosphorus pentoxide was then added to the chitosan solution, at $0.1,0.5,1$ and 2 equivalent moles to the chitosan residue. The reaction was allowed to proceed for $3 \mathrm{~h}$ in an ice bath at $0-5{ }^{\circ} \mathrm{C}$. The mixture of chitosan solution with phosphorus pentoxide after $3 \mathrm{~h}$ of reaction was poured into a large amount of acetone and washed with acetone several times. The phosphorylated chitosan was then dried in an oven at $40{ }^{\circ} \mathrm{C}$ for around $5 \mathrm{~h}$.

\section{Elimination of inter- and intramolecular crosslinks by sodium salt formation}

The dried powder of phosphorylated chitosan was dissolved in distilled water Both the water-soluble product and the insoluble product, which was attributed to the formation of inter- or intramolecular crosslinks, were preliminarily dialyzed against distilled water for 2 days to remove unreacted phosphorus pentoxide. To eliminate the inter- and intramolecular crosslinks of amino groups-methanesulfonic acid salt with phosphoric groups, the sodium salt of phosphorylated chitosan or sodium phosphorylated chitosan was prepared through sodium salt formation by further dialysis in $0.1 \mathrm{~m}$ sodium hydroxide solution for $12 \mathrm{~h}$. The $\mathrm{pH}$ of the resulting solution was adjusted to neutral with $0.1 \mathrm{~m}$ hydrochloric acid and then dialyzed against distilled water for 2 days to remove the sodium chloride by-product. The remaining water-insoluble product was filtered from the solution. The white sponge of sodium phosphorylated chitosan was obtained by freeze drying. The percentage yields of the water-soluble product and water-insoluble product, as well as the normalized percentage yield, were calculated as follows:

$$
\% \text { Yield }=\frac{W_{\mathrm{p}}}{\left(W_{\mathrm{cts}}+W_{\text {phos }}\right)} \times 100
$$

where $W_{\mathrm{p}}=$ dried weight of the obtained products (water-soluble product or water-insoluble product), $W_{\mathrm{cts}}=$ dried weight of chitosan used in the reaction, $W_{\text {phos }}=$ dried weight of phosphorus pentoxide added in the reaction.

The normalized percentage yield was calculated by comparing the weight of the obtained products (water-soluble or water-insoluble products) with the dried weight of the total products:

$$
\begin{aligned}
& \text { Normalized } \% \text { yield }_{\text {sol }}=\frac{W_{\text {sol }}}{\left(W_{\text {sol }}+W_{\text {insol }}\right)} \times 100 \\
& \text { Normalized } \% \text { yield }_{\text {insol }}=\frac{W_{\text {insol }}}{\left(W_{\text {sol }}+W_{\text {insol }}\right)} \times 100
\end{aligned}
$$

where $W_{\text {sol }}=$ dried weight of the water-soluble product, $W_{\text {insol }}=$ dried weight of the water-insoluble product.

\section{Structural characterization}

The structure of the obtained chitosan derivatives was analyzed by FT-IR using Model Spectrum One from Perkin Elmer (Shelton, CT, USA). The KBr pellet was prepared using a sample-to- $\mathrm{KBr}$ ratio of $1 / 100$. FT-IR spectra were scanned from $4000-400 \mathrm{~cm}^{-1}$, with a resolution of $4.0 \mathrm{~cm}^{-1}$ using a DLATGS detector (Thermo Scientific, West Haven, CT, USA); the number of scans was 16.

The nuclear magnetic resonance (NMR) spectra of sodium phosphorylated chitosan were analyzed using an Advance $300 \mathrm{MHz}$ Digital NMR spectrometer, Model DPX 300, Bruker Biospin (Billerica, MA, USA) by the solid-state ${ }^{13} \mathrm{C}$ CP/MAS NMR technique. NMR spectra were recorded at a frequency of $75 \mathrm{MHz}$ and were scanned with 1600 scans, a relaxation delay of $4 \mathrm{~s}$, a spin rate of $5 \mathrm{kHz}$ and spectra sizes of $2 \mathrm{~K}$ with a $4 \mathrm{~K}$ time domain size.

\section{Degree of phosphoric substitution}

The DS was determined using a SEM, JSM-5800, JEOL (Tokyo, Japan) equipped with an energy-dispersive X-ray spectrometer. Chitosan derivative samples were ground and placed on SEM carbon holders with carbon tape. SEM images were viewed under pressure with a voltage of $20 \mathrm{kV}$ and a magnification of 2500. An electron beam was set at a constant distance of $15 \mu \mathrm{m}$. The percentage of elements present in samples was recorded. The degree of phosphoric substitution to chitosan can be calculated using the following equation:

$$
\text { Degree of substitution }=\frac{\% P}{\% N}
$$

where $\% P=$ phosphorus content of phosphoric groups, $\% N=$ nitrogen content in chitosan molecules.

\section{Solubility test}

The solubility of chitosan derivatives was evaluated by dissolving $2 \mathrm{mg}$ of the sample into $2 \mathrm{ml}$ of three kinds of solvents: $0.1 \mathrm{M}$ acetic acid $\left(\mathrm{pH} \mathrm{3.4,30}{ }^{\circ} \mathrm{C}\right)$, $0.1 \mathrm{~m}$ sodium hydrogen carbonate $\left(\mathrm{pH} 8.6,30^{\circ} \mathrm{C}\right)$ and distilled water $(\mathrm{pH} 6.6$, $\left.30{ }^{\circ} \mathrm{C}\right)$

\section{Enhancement of the elimination of inter- and intramolecular crosslinks by washing solvent selection, followed by sodium salt formation}

The formation of inter- or intramolecular crosslinks was considered to occur between protonated amino groups and phosphoric groups. Methanesulfonic acid was expected to be the main cause of protonated amino groups in chitosan, after the formation of inter- or intramolecular crosslinks between protonated amino groups (ammonium groups) and phosphoric groups. To enhance the elimination of inter- and intramolecular crosslinks, ethanol was selected as an alternative to acetone to eliminate the amino groups-methanesulfonic acid salt formation. Phosphorylated chitosan with 2 equivalent moles of phosphorus pentoxide to chitosan residue was synthesized as above. The mixture was then precipitated in a large amount of acetone and washed several times with ethanol instead. The phosphorylated chitosan was then dried in an oven at $40{ }^{\circ} \mathrm{C}$ for around $5 \mathrm{~h}$. Thereafter, sodium salt formation was carried out as mentioned above to eliminate the inter- and intramolecular crosslinks between ammonium groups and phosphoric groups.

\section{RESULTS AND DISCUSSION}

\section{Synthesis of phosphorylated chitosan}

Phosphorylated chitosan was synthesized by the reaction of chitosan with phosphorus pentoxide in methanesulfonic acid. The degree of phosphoric substitution was varied by varying the phosphorus pentoxide to chitosan residue in the range of 0.1-2 equivalent moles. Methanesulfonic acid was used as the protective agent for the amino groups of chitosan. ${ }^{21}$ Therefore, the substitution of phosphoric groups was allowed to occur at the C-3 and C-6 hydroxyl groups of chitosan. Phosphorylated chitosan was obtained by precipitation in acetone; it was then washed with acetone several times and dried at $40^{\circ} \mathrm{C}$. The obtained phosphorylated chitosan was then dissolved in distilled water and dialyzed against distilled water for 2 days to remove the unreacted phosphorus pentoxide. A larger amount of insoluble phosphorylated chitosan was obtained with a larger amount of phosphorus pentoxide, as a consequence of a higher degree of phosphoric substitution. Nishi et al. ${ }^{20,21}$ varied the degree of phosphoric substitution to chitin or $45 \%$ deacetylated chitin and found that chitin phosphate can be dissolved in water independently of the DS, whereas water solubility of deacetylated chitin phosphate decreased as the DS increased. The water solubility of deacetylated chitin phosphate or chitosan phosphate was inversely proportional not only to the degree of phosphoric substitution ${ }^{20,21}$ but also to the degree of deacetylation (DD). ${ }^{9}$ Therefore, phosphorylation with a high DD chitin, that is, chitosan, tends to yield water-insoluble phosphorylated chitosan. The insolubility of 
phosphorylated chitosan with a high degree of phosphoric substitution was attributed to inter- and intramolecular crosslinks.

Elimination of inter- and intramolecular crosslinks by sodium salt formation

As inter- and intramolecular crosslinks are caused by the electrostatic interaction between ammonium groups and phosphoric groups, amino groups were protonated by methanesulfonic acid in a salt form. Therefore, an alkaline condition was considered to destroy this electrostatic interaction, concomitantly eliminating inter- and intramolecular crosslinks, as amino groups could not be ionized under alkaline conditions. Sodium salt formation destroys the electrostatic interaction between ammonium groups and phosphoric groups, which neutralizes methanesulfonic acid. Sodium salt formation of phosphorylated chitosan was carried out by dialyzing both watersoluble and water-insoluble phosphorylated chitosan against $0.1 \mathrm{M}$ sodium hydroxide for $12 \mathrm{~h}$. The mixture with excess sodium hydroxide was neutralized with $0.1 \mathrm{~m}$ hydrochloric acid. After neutralization, the obtained mixture was dialyzed again against distilled water for 2 days to remove sodium chloride. The white product of sodium-phosphorylated chitosan was obtained after freeze drying. Salts of alkaline metals show good solubility in water. Thus, sodium-phosphorylated chitosan was thoroughly dissolved in distilled water. A schematic synthesis pathway is shown in Figure 1. When using phosphorus pentoxide at
0.1 equivalent moles to chitosan residue, the obtained sodium phosphorylated chitosan was totally soluble in water. With phosphorus pentoxide at $0.5-1$ equivalent moles to chitosan residue, a black brown precipitant of phosphorylated chitosan was obtained. The black brown color is the same as that of chitosan dissolved in methanesulfonic acid, implying that methanesulfonic acid still remained within the phosphorylated chitosan. When dissolving phosphorylated chitosan in distilled water, the $\mathrm{pH}$ of the solution changed to approximately 3 , resulting in completely dissolved phosphorylated chitosan. Next, sodium salt formation and neutralization were allowed to continue to synthesize sodium phosphorylated chitosan. Surprisingly, a certain amount of sodium phosphorylated chitosan was reversed to phosphorylated chitosan and precipitated during neutralization with $0.1 \mathrm{~m}$ hydrochloric acid. Therefore, water-soluble sodium phosphorylated chitosan and water-insoluble phosphorylated chitosan were obtained after neutralization. This phenomenon indicated that the sodium salt of phosphorylated chitosan has a higher ability to dissolve in water than phosphorylated chitosan. Using phosphorus pentoxide at 2 equivalent moles to chitosan residue, the black brown product of phosphorylated chitosan was similarly obtained after phosphorylation, implying that methanesulfonic acid still remained in the phosphorylated chitosan. The $\mathrm{pH}$ of phosphorylated chitosan that dissolved in water was $\sim 3$. However, only a small amount of phosphorylated chitosan could be dissolved in water with continued
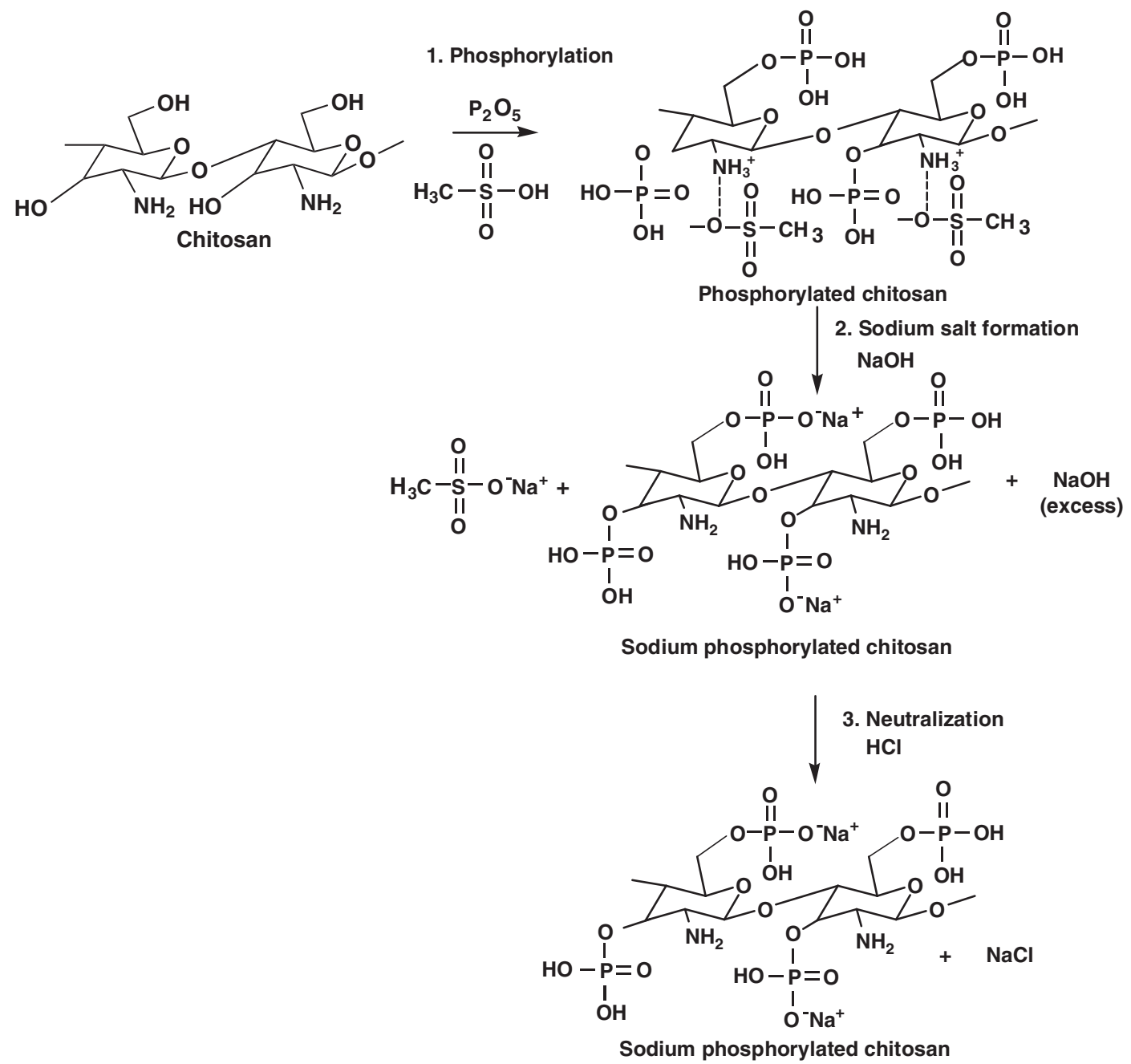

Figure 1 The synthesis pathway of sodium phosphorylated chitosan. 


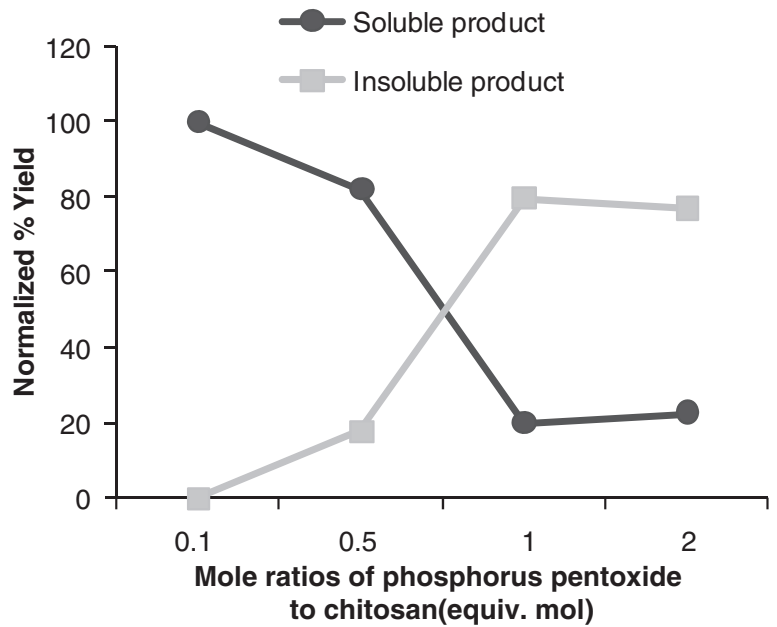

Figure 2 Normalized percentage yield of sodium phosphorylated chitosan (water-soluble product) and phosphorylated chitosan (water-insoluble product) compared with their total percentage yield.

sodium salt formation and neutralization to obtain sodium phosphorylated chitosan. Most of the phosphorylated chitosan, however, could not be dissolved and formed a sticky black brown product floating on the surface of the water. The insolubility of phosphorylated chitosan synthesized with phosphorus pentoxide at 2 equivalent moles to chitosan residue is attributed to the formation of inter- or intramolecular crosslinks between amino groups-methanesulfonic acid salt and the substituted phosphoric groups. These phenomena imply that methanesulfonic acid protonated the amino groups to crosslink with phosphoric groups by an electrostatic interaction. Therefore, inter- or intramolecular crosslinks should be stabilized to the level of high phosphoric substitution.

The normalized percentage yields of water-soluble sodium phosphorylated chitosan and water-insoluble phosphorylated chitosan are described in Figure 2. This figure indicates that the normalized percentage yield of water-soluble sodium phosphorylated chitosan decreased as the mole ratio of phosphorus pentoxide to chitosan residue increased. By contrast, the normalized percentage yield of water-insoluble phosphorylated chitosan increased as the mole ratios of phosphorus pentoxide to chitosan residue increased. The normalized percentage yield of water-insoluble phosphorylated chitosan synthesized with 2 equivalent moles phosphorus pentoxide, as shown in Figure 2, was the percentage yield before sodium salt formation. In addition, sodium salt formation was carried out in another experiment for inter- and intramolecular crosslinked phosphorylated chitosan. It was found that a large amount of insoluble phosphorylated chitosan was altered to a soluble sodium salt of phosphorylated chitosan. However, a small amount of insoluble product remained. The percentage yield was not calculated in this experiment.

\section{Structural characterization}

Chitosan, a water-insoluble product of phosphorylated chitosan, and water-soluble products of sodium phosphorylated chitosan were structurally characterized by FT-IR. Figure 3 presents the FT-IR spectra of chitosan and water-soluble sodium phosphorylated chitosan synthesized from different mole ratios of phosphorus pentoxide to chitosan residue. Using FT-IR $\left(\mathrm{KBr}, \mathrm{cm}^{-1}\right)$, chitosan shows characteristic peaks as follows: 3426 (OH stretching and N-H stretching), 2932 (C-H stretching of methyl), 1652 ( $\mathrm{C}=\mathrm{O}$ stretching), 1596 (N-H bending) and 1078 (C-O stretching of pyranose ring). Sodium phosphorylated chitosan presents the same peaks as chitosan and new absorption wave numbers $\left(\mathrm{cm}^{-1}\right)$, which are the characteristic peaks of phosphoric groups appearing at $1240(\mathrm{P}=\mathrm{O}$ stretching $), 975$ $(\mathrm{P}-\mathrm{OH})$ and $800(\mathrm{P}-\mathrm{O}-\mathrm{C})$. The absorption peaks at 1240 and $800 \mathrm{~cm}^{-1}$ tend to increase as the mole ratios of phosphorus pentoxide to chitosan residue increase, resulting in an increased substitution of phosphoric groups to hydroxyl groups of chitosan at the C-3 and C-6 positions. The hydroxyl groups at the C-3 and C-6 positions could be equally substituted with phosphoric groups. ${ }^{20}$ Figure 4 shows the FT-IR spectra of the water-insoluble products of phosphorylated chitosan synthesized from different mole ratios of phosphorus pentoxide to chitosan residue. Phosphorylated chitosan shows the same characteristic peaks of phosphoric substitution as water-soluble sodium phosphorylated chitosan, indicating the same functional structure but differing solely by phosphoric substitution and the partial sodium salt of phosphoric substitution. Interestingly, the additional absorption peak at $1175 \mathrm{~cm}^{-1}$, which represents S-O stretching, was observed in the FT-IR spectrum of phosphorylated chitosan synthesized from 2 equivalent moles of phosphorus pentoxide to chitosan residue, indicating that the methanesulfonic acid included with phosphorylated chitosan was difficult to remove with acetone, resulting in inter- or intramolecular crosslinks.

\section{Degree of phosphoric substitution}

The degrees of substitution of phosphorylated chitosan and sodiumphosphorylated chitosan were determined by SEM equipped with energy-dispersive X-ray spectrometer, as shown in Table 1. The degrees of substitution of phosphorylated chitosan and sodium phosphorylated chitosan show the same upward trend as the mole ratios of phosphorus pentoxide to chitosan residue increase. In addition, it was found that the DS of sodium phosphorylated chitosan was almost the same as that of phosphorylated chitosan at each equivalent moles ratio. Both phosphorylated chitosan and sodium phosphorylated chitosan possess the same functional structure but differ solely in terms of phosphoric acid substitution and the partial sodium salt of phosphoric acid substitution, respectively.

\section{Solubility test}

The solubility of phosphorylated chitosan and sodium phosphorylated chitosan was tested in three kinds of solvents, including $0.1 \mathrm{M}$ acetic acid, $0.1 \mathrm{~m}$ sodium hydrogen carbonate and distilled water. The solubility results for the three solvents are summarized in Table 1. It was found that sodium phosphorylated chitosan (DS 0.01) can be dissolved in dilute acid in a manner similar to that of the original chitosan. In addition, its water solubility was also developed. On the other hand, sodium phosphorylated chitosan (DS 0.5-0.6) can be dissolved in alkaline conditions because of the negative charges of phosphoric groups. Phosphorylated chitosan, even with a high DS (DS 0.61 ), cannot be dissolved under either acidic or alkaline conditions. This finding is attributed to the large amount of negative phosphoric groups concomitantly forming inter- or intramolecular crosslinks with ammonium groups. Interestingly, both sodium phosphorylated chitosan and phosphorylated chitosan of medium DS (DS 0.05-0.3) were found to dissolve under both acidic and alkaline conditions, representing amphoteric properties. It is interesting to note that phosphorylated chitin is easily soluble in water, independent of DS. ${ }^{20}$ In contrast, the water solubility of phosphorylated chitosan depends on DS, as well as on the DD. High DD chitosan has a large number of amino groups to be protonated by methanesulfonic acid and therefore forms inter- or intramolecular crosslinks that are the main cause of insolubility. 


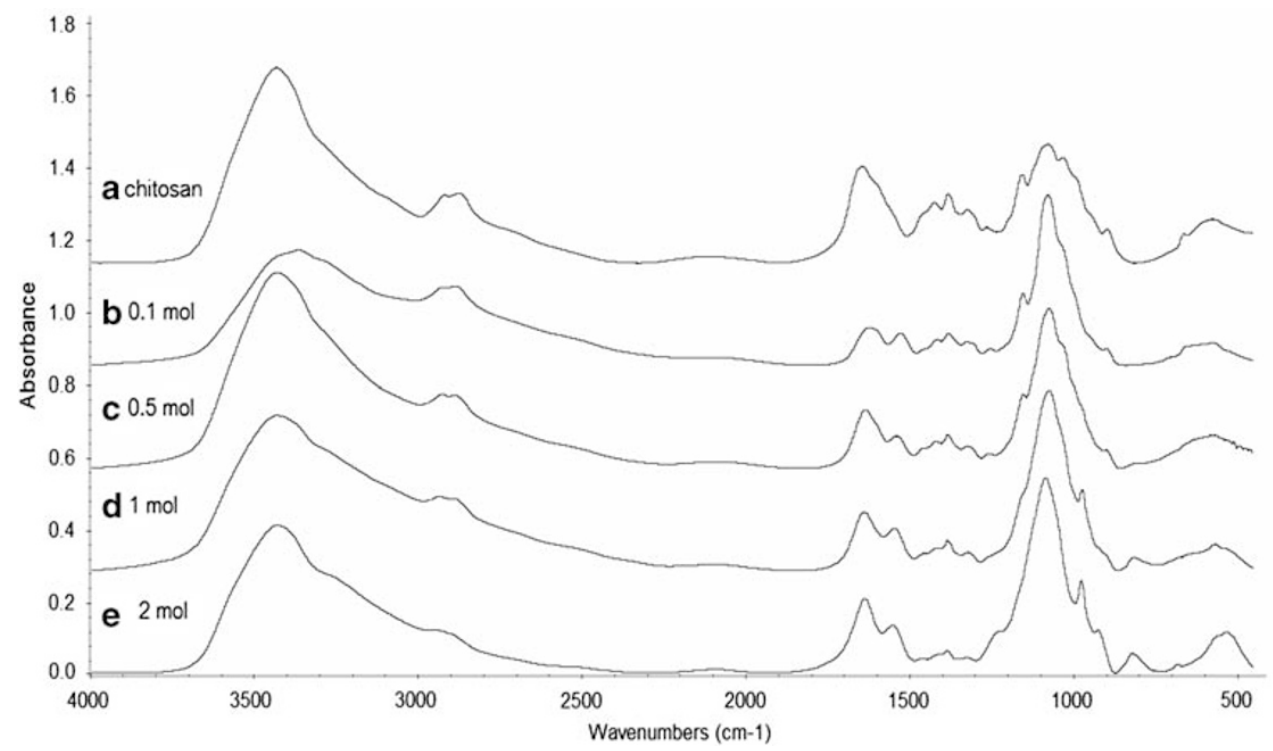

Figure 3 Fourier transform infrared spectra of (a) chitosan and sodium phosphorylated chitosan (water-soluble products) synthesized from different mole ratios of phosphorus pentoxide to chitosan residue of (b) 0.1 , (c) 0.5 , (d) 1 and (e) 2 equivalent moles.

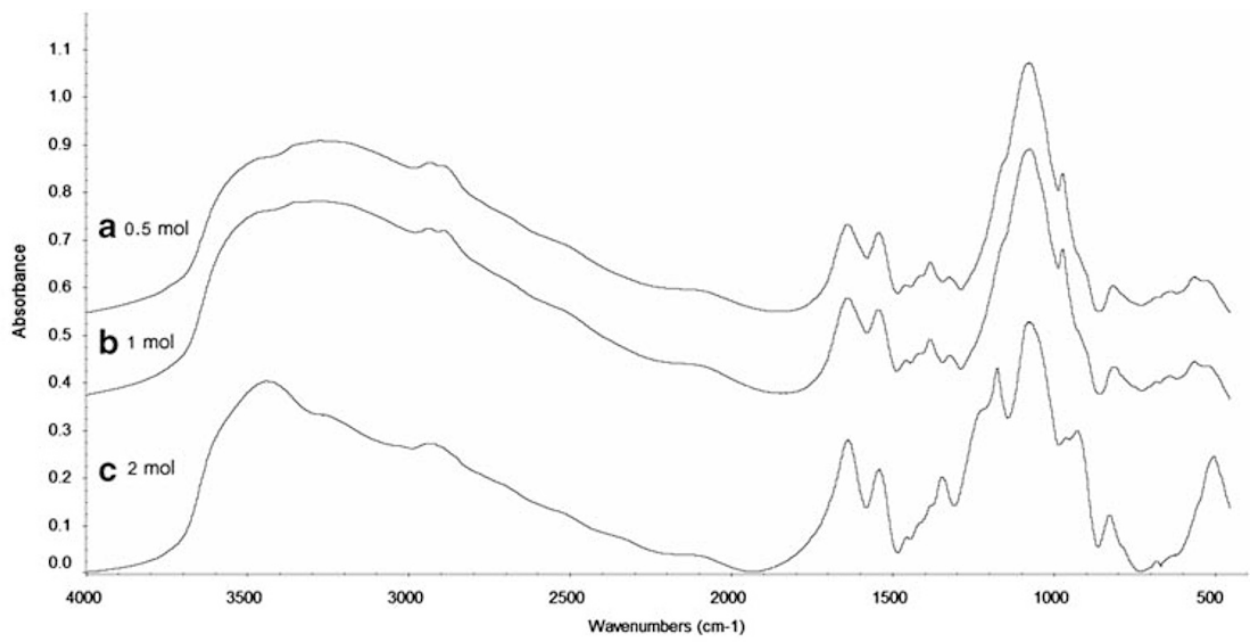

Figure 4 Fourier transform infrared spectra of phosphorylated chitosan (water-insoluble products) synthesized from different mole ratios of phosphorus pentoxide to chitosan residue of (a) 0.5 , (b) 1 and (c) 2 equivalent moles.

Table 1 Solubility test and degree of phosphoric substitution of sodium phosphorylated chitosan and phosphorylated chitosan

\begin{tabular}{|c|c|c|c|c|c|c|c|c|}
\hline $\begin{array}{l}\text { Mole ratios of phosphorus pentoxide to chitosan } \\
\text { residue (equivalent moles) }\end{array}$ & $D S^{a}$ & Base $^{\mathrm{b}}$ & Water & Acid $^{\mathrm{d}}$ & $D S^{\mathrm{a}}$ & Base $^{b}$ & Water & Acid $^{\mathrm{d}}$ \\
\hline 0.5 & 0.05 & 0 & 0 & 0 & 0.18 & 0 & $x$ & $\Delta$ \\
\hline 1 & 0.3 & 0 & 0 & 0 & 0.3 & 0 & $x$ & 0 \\
\hline 2 & 0.5 & 0 & 0 & $x$ & 0.61 & $x$ & $x$ & $x$ \\
\hline
\end{tabular}

Abbreviations: 0 , Soluble; $X$, insoluble; $\triangle$, partially soluble.

aDetermined by scanning electron microscope equipped with an energy-dispersive $\mathrm{X}$-ray spectrometer.

b $0.1 \mathrm{M}$ Sodium hydrogen carbonate, $\mathrm{pH} 8.6$.

cDistilled water, $\mathrm{pH} 6.6$

do.1 M Acetic acid, pH 3.4 
Enhancement of the elimination of inter- and intramolecular crosslinks by washing with ethanol, followed by sodium salt formation

The formation of inter- and intramolecular crosslinks was considered to occur between ammonium groups and phosphoric groups. Amino groups induced methanesulfonic acid to surround the molecules and stabilized them with phosphoric groups by electrostatic interaction. Therefore, inter- or intramolecular crosslinks are as stabilized as the degree of phosphoric group substitution. Therefore, methanesulfonic acid was considered to be the main factor involving inter- and intramolecular crosslinks. Elimination of inter- and intramolecular crosslinks should be carried out by avoiding the salt form of protonated amino groups with methanesulfonic acid. Owing to the fact that methanesulfonic acid protonates the amino groups and forms salt complexes by electrostatic interaction, washing with acetone is not sufficient to interfere with the amino groups-methanesulfonic acid salt. Ethanol was selected as an alternative washing solvent to avoid the salt formation of amino groups-methanesulfonic acid. The sodium phosphorylated chitosan with 2 equivalent moles of phosphorus pentoxide to chitosan residue was synthesized as formerly described. Ethanol was later used to wash phosphorylated chitosan several times instead of acetone, followed by drying in the oven at $40{ }^{\circ} \mathrm{C}$ for around $5 \mathrm{~h}$. Surprisingly, after being washed with ethanol, phosphorylated chitosan could be dissolved in distilled water completely, whereas after being washed with acetone, phosphorylated chitosan yielded a sticky black brown product that floated on the surface of the distilled water, which was attributed to inter- or intramolecular crosslinks. This phenomenon indicated that ethanol is a potential solvent that helps to avoid the salt formation of amino groups-methanesulfonic acid. However, the $\mathrm{pH}$ of the solution still remains $\sim 3$ when dissolving phosphorylated chitosan in distilled water, indicating the existence of methanesulfonic acid. Sodium salt formation was used to eliminate inter- and intramolecular crosslinks caused by the electrostatic interaction between protonated amino groups and phosphoric groups. The effect of sodium salt formation is not only to eliminate inter- and intramolecular crosslinks but also to neutralize methanesulfonic acid. The percentage yield of sodium phosphorylated chitosan using ethanol washing compared with that of sodium phosphorylated chitosan using acetone washing is summarized in Table 2. The total percentage yield of sodium phosphorylated chitosan washed with ethanol is close to the total percentage yield of sodium phosphorylated chitosan washed with acetone plus inter- or intramolecular crosslinked phosphorylated chitosan. Sodium phosphorylated chitosan can be synthesized here at a low percentage yield. As the glycosidic linkage is generally labile to acid, a decrease in molecular weight occurred during the reaction in methanesulfonic acid. The low molecular weight of chitosan derivatives (determined by high-performance liquid chromatography, data not shown) may not be reprecipitated in acetone or dialyzed throughout MWCO 6000-8000, resulting in a low percentage yield. The phosphate ester of polysaccharides was shown to be remarkably stable during base-catalyzed hydrolysis. ${ }^{23}$

Figure 5 shows the FT-IR spectra of sodium phosphorylated chitosan washed with acetone and ethanol. The acetone-washed sodium phosphorylated chitosan and ethanol-washed sodium phos-

Table $2 \%$ Yield of sodium phosphorylated chitosan and phosphorylated chitosan washing with acetone or ethanol

\begin{tabular}{|c|c|c|c|c|c|c|}
\hline \multirow[b]{2}{*}{$\begin{array}{l}\text { Mole ratio of phosphorus pentoxide to } \\
\text { chitosan residue (equivalent moles) }\end{array}$} & \multirow[b]{2}{*}{$\begin{array}{l}\text { Washing } \\
\text { solvents }\end{array}$} & \multicolumn{4}{|c|}{$\%$ Yield } & \multirow[b]{2}{*}{$\begin{array}{l}\text { Total } \\
\text { yield }{ }^{\mathrm{b}}(\%)\end{array}$} \\
\hline & & $D S^{a}$ & $\begin{array}{l}\text { Sodium phosphorylated } \\
\text { chitosan (water-soluble) }\end{array}$ & $D S^{a}$ & $\begin{array}{c}\text { Inter- and intramolecular crosslinked } \\
\text { phosphorylated chitosan (water-insoluble) }\end{array}$ & \\
\hline 2 & Acetone & 0.5 & 5.29 & 0.61 & 17.8 & 23.09 \\
\hline 2 & Ethanol & 0.53 & 27.71 & & - & 27.71 \\
\hline
\end{tabular}

aDetermined by scanning electron microscope equipped with an energy-dispersive $\mathrm{X}$-ray spectrometer.

b\% Yield of water-soluble product $+\%$ yield of water-insoluble product.

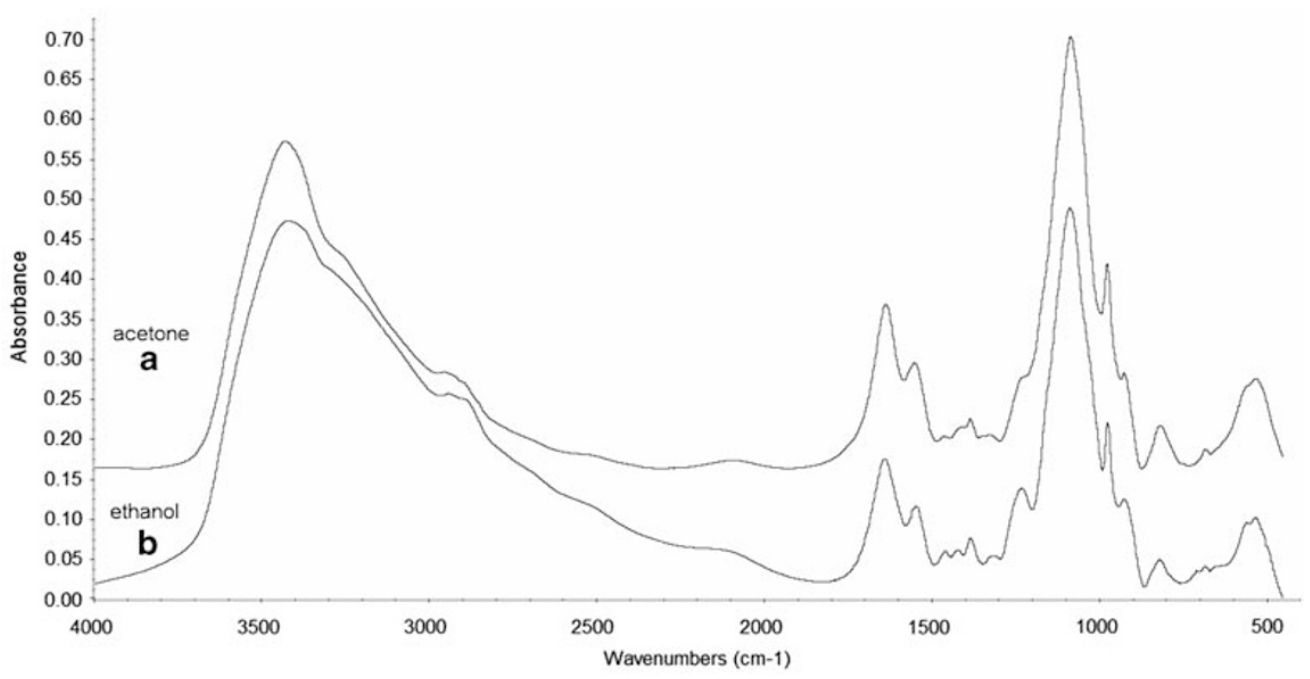

Figure 5 Fourier transform infrared spectra of sodium phosphorylated chitosan (water-soluble products) synthesized with a mole ratio of 2 equivalent moles of phosphorus pentoxide to chitosan residue of, using washing solvents (a) acetone and (b) ethanol. 
phorylated chitosan represent the same characteristic peaks of phosphoric groups appearing at $1240 \mathrm{~cm}^{-1}\left(\mathrm{P}=\mathrm{O}\right.$ stretching), $975 \mathrm{~cm}^{-1}$ $(\mathrm{P}-\mathrm{OH})$ and $800 \mathrm{~cm}^{-1}$ (P-O-C). Next, the degree of phosphoric substitution of the sodium phosphorylated chitosan washed with ethanol was examined by SEM equipped with energy-dispersive Xray spectrometer. The DS (Table 2) of ethanol-washed sodium phosphorylated chitosan was 0.53 , close to the DS of acetone-washed sodium phosphorylated chitosan (which was 0.50 ). Therefore, ethanol washing does not influence the chemical structure or DS of sodium phosphorylated chitosan; however, it influences the electrostatic interaction of amino groups and methanesulfonic acid. The subsequent sodium salt formation to eliminate inter- or intramolecular crosslinks between ammonium groups and phosphoric groups resulted in a completely water-soluble sodium phosphorylated chitosan, independent of the degree of phosphoric substitution.

The NMR spectra of sodium phosphorylated chitosan were determined using solid-state cross-polarization/magic angle spinning (CP/ MAS ${ }^{13} \mathrm{C}$ NMR), using chitosan as a control. The ${ }^{13} \mathrm{C}$ signals of chitosan (Figure 6a) were C1 ( $\delta 106.29$ p.p.m.), C2 ( $\delta 57.75$ p.p.m.), C3 and C5 ( $\delta 75.40$ p.p.m.), C4 ( $\delta 83.15$ p.p.m.), C6 ( $\delta 60.68$ p.p.m.), $\mathrm{C}=\mathrm{O}(\delta 174.45$ p.p.m. $)$ and $\mathrm{CH}_{3}(\delta 23.74$ p.p.m. $)$. It is noteworthy that $\mathrm{C} 3$ and $\mathrm{C} 5$ tend to form one single resonance in $\beta$-chitin. ${ }^{24}$ The ${ }^{13} \mathrm{C}$ signals of sodium phosphorylated chitosan (Figure $\left.6 \mathrm{~b}\right)$ were $\mathrm{C} 1(\delta$ 100.94 p.p.m.), C2 ( $\delta 56.82$ p.p.m.), C3 and C5 ( $\delta 74.64$ p.p.m.), C4 $(\delta 81.00$ p.p.m.), C6 ( $\delta 62.65$ p.p.m.), $\mathrm{C}=\mathrm{O}(\delta 175.73$ p.p.m. $)$ and $\mathrm{CH}_{3}$ ( $\delta$ 23.95 p.p.m.). The intensity of the chemical shift for C6 decreased as a result of phosphoric substitution. The chemical shift for C3 changed slightly because of phosphoric substitution. It can also be observed that the intensity of the chemical shift for C4 decreased, probably because of the effect of substitution of phosphoric groups at the neighboring $\mathrm{C} 3$ position. This result indicates that phosphoric substitution occurred at C6 and C3 equally.

It is also noteworthy that the insolubility of phosphorylated chitosan was involved in the formation of inter- and intramolecular crosslinks. Two main factors corresponding to the formation of interand intramolecular crosslinks include (i) the electrostatic interaction
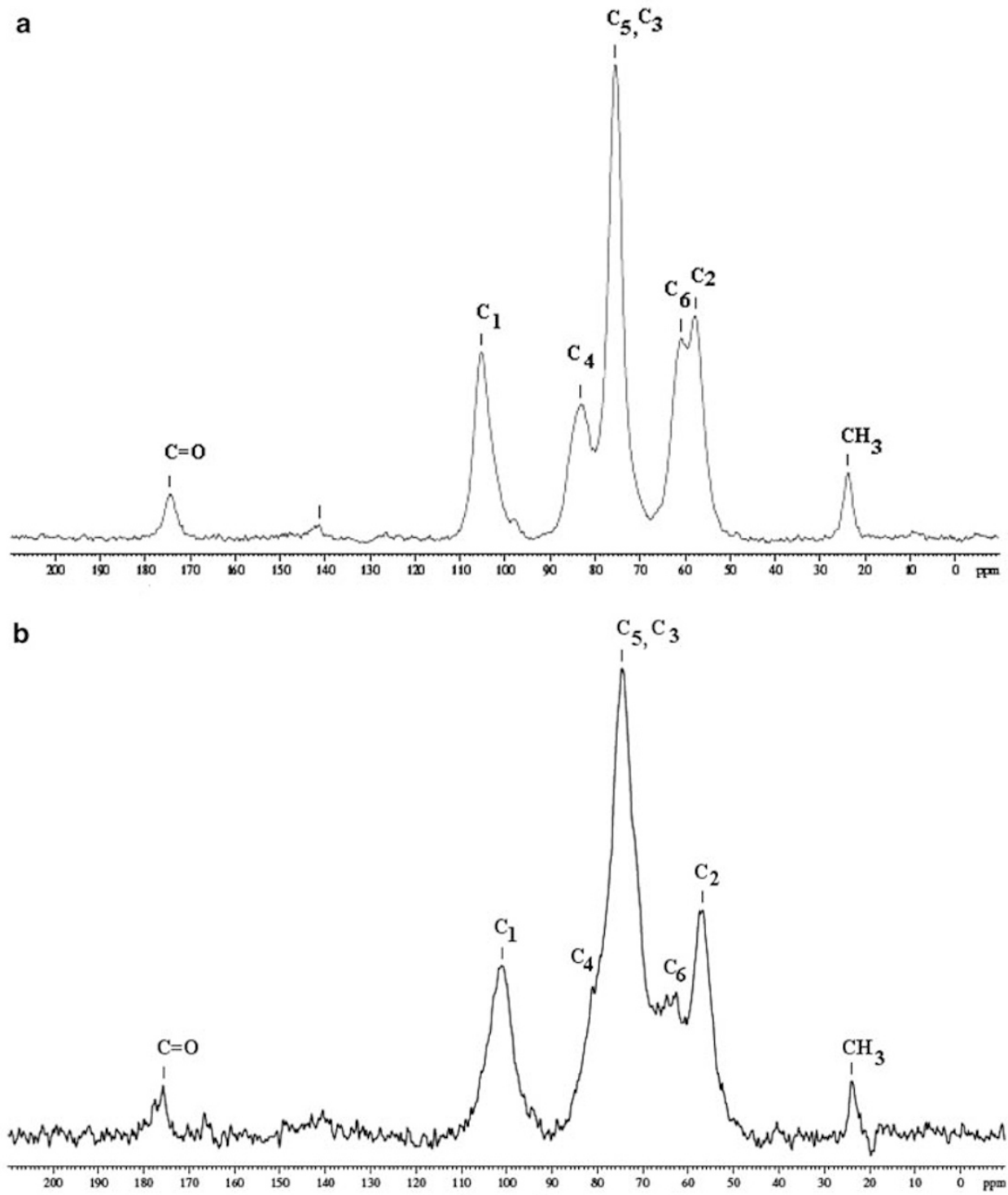

Figure $6{ }^{13} \mathrm{C}$ solid-state NMR spectra of (a) chitosan and (b) sodium phosphorylated chitosan. 


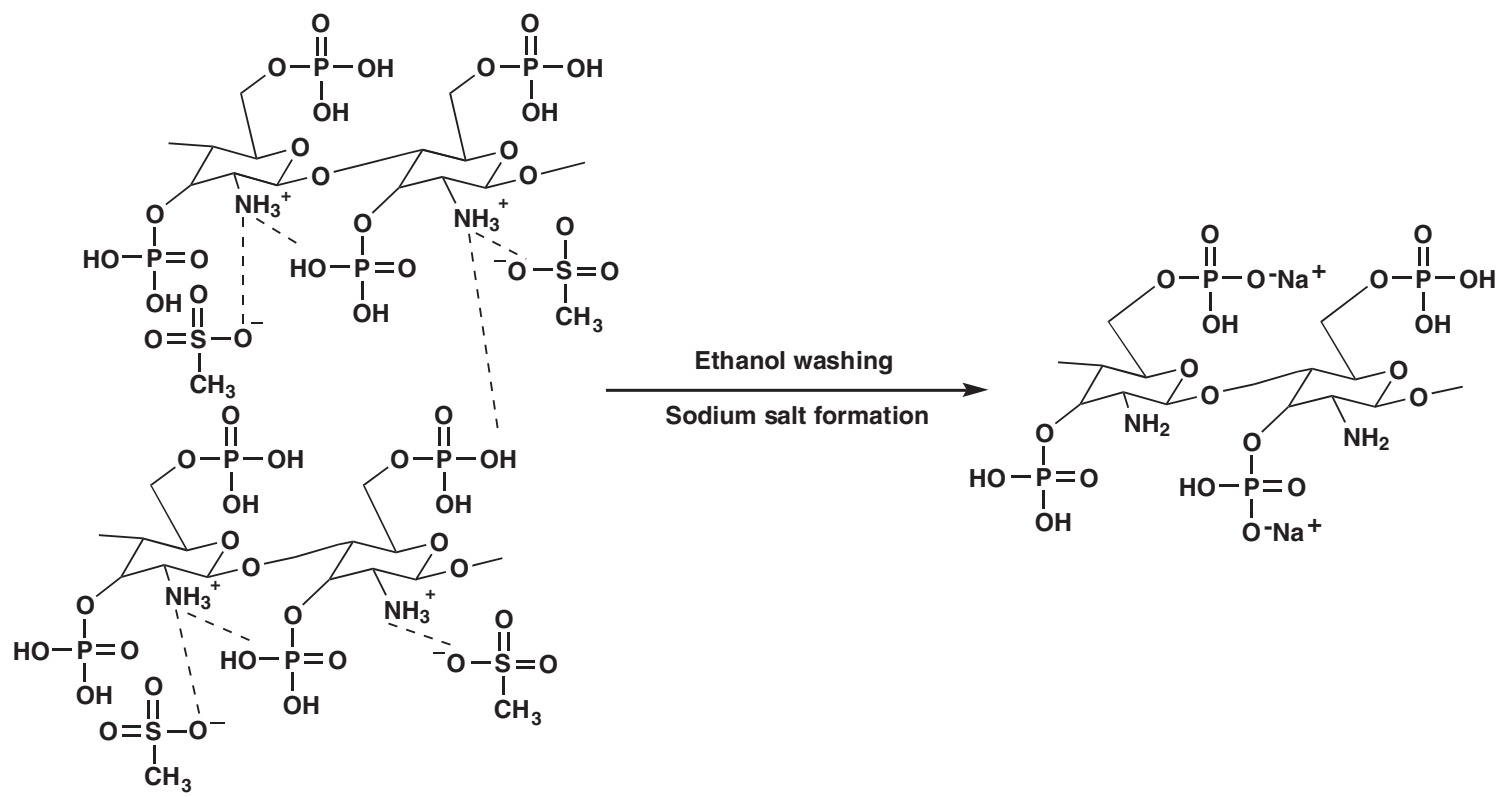

Figure 7 Elimination of inter- and intramolecular crosslinks by ethanol washing after phosphorylation, followed by sodium salt formation.

between amino groups and methanesulfonic acid salt formation and (ii) the electrostatic interaction between ammonium groups and phosphoric groups. To synthesize water-soluble phosphorylated chitosan, ethanol washing after phosphorylation, followed by sodium salt formation, is considered as a potential method. A schematic diagram of the elimination of inter- and intramolecular crosslinks is summarized in Figure 7.

\section{CONCLUSIONS}

The insolubility of phosphorylated chitosan synthesized through the phosphorus pentoxide/methanesulfonic acid system mainly results from the formation of inter- and intramolecular crosslinks. Two main factors for the formation of inter- and intramolecular crosslink are (i) electrostatic interaction between amino groups and methanesulfonic acid salt formation and (ii) electrostatic interaction between ammonium groups and phosphoric groups. Ethanol washing interfered with the electrostatic interaction between amino groups and methanesulfonic acid salt. Sodium salt formation interfered with the electrostatic interaction between ammonium groups and phosphoric groups. Therefore, water-soluble sodium phosphorylated chitosan was successfully synthesized by phosphorylation in a phosphorus pentoxide/methanesulfonic acid system through washing with ethanol, followed by sodium salt formation. Water solubility was independent of the degree of phosphoric substitution.

\section{ACKNOWLEDGEMENTS}

This project was supported by Grants for Development of New Faculty Staff, Chulalongkorn University and partially by the Graduate Thesis Grant, Chulalongkorn University.

1 Fangkangwanwong, J., Akashi, M., Kida, T. \& Chirachanchai, S. One-pot synthesis in aqueous system for water-soluble chitosan-graft-poly(ethylene glycol) methyl ether. Biopolymers 82, 580-586 (2006).

2 Pasanphan, W. \& Chirachanchai, S. Conjugation of gallic acid onto chitosan: an approach for green and water-based antioxidant. Carbohydr. Polym. 72, 169-177 (2008).
3 Gomes, P., Gomes, C. A. R., Batista, M. K. S., Pinto, L. F. \& Silva, P. A. P. Synthesis, structural characterization and properties of water-soluble $\mathrm{N}$-( $\gamma$-propanoyl-amino acid)chitosans. Carbohydr. Polym. 71, 54-65 (2008).

4 Sashiwa, H. \& Shigemasa, Y. Chemical modification of chitin and chitosan 2: preparation and water soluble property of $\mathrm{N}$-acylated or $\mathrm{N}$-alkylated partially deacetylated chitins. Carbohydr. Polym. 39, 127-138 (1999).

5 Zhang, C., Ping, Q., Zhang, H. \& Shen, J. Synthesis and characterization of watersoluble O-succinyl-chitosan. Eur. Polym. J. 39, 1629-1634 (2003).

6 Singh, J., Dutta, P. K., Dutta, J., Hunt, A. J., Macquarrie, D. J. \& Clark, J. H. Preparation and properties of highly soluble chitosan-L-glutamic acid aerogel derivative. Carbohydr. Polym. 76, 188-195 (2009).

7 Wang, X., Ma, J., Wang, Y. \& He, B. Bone repair in radii and tibias of rabbits with phosphorylated chitosan reinforced calcium phosphate cements. Biomaterials 23, 4167-4176 (2002).

8 Wang, X., Ma, J., Feng, Q. \& Cui, F. Skeletal repair in rabbits with calcium phosphate cements incorporated phosphorylated chitin. Biomaterials 23, 4591-4600 (2002).

9 Wang, X., Ma, J., Wang, Y. \& He, B. Structural characterization of phosphorylated chitosan and their applications as effective additives of calcium phosphate cements. Biomaterials 22, 2247-2255 (2001).

10 Zhu, Y., Wang, X., Cui, F. Z. \& Feng, Q. L. In vitro cytocompatibility and osteroinductive of phosphorylated chitosan with osteoblasts. J. Bioact. Compat. Polym. 18, 375-390 (2003).

11 Varma, H. K., Yokogawa, Y., Espinosa, F. F., Kawamoto, Y., Nishizawa, K., Nagata, F. \& Kameyama, T. Porous calcium phosphate coating over phosphorylated chitosan film by a biomimetic method. Biomaterials 20, 879-884 (1999).

12 Li, Q. L., Chen, Z. Q., Barvell, B. W., Liu, L. K., Jiang, H. B., Zen, Q., Peng, Q. \& Ou, G. M. Chitosan-phosphorylated chitosan polyelectrolyte complex hydrogel as an osteoblast carrier. J. Biomed. Mater. Res. 82B, 481-486 (2007).

13 Li, Q. L., Wu, M. Y., Tang, L. L., Zhou, J. \& Jiang, Y. Bioactivity of a novel nanocomposite of hydroxyapatite and chitosan-phosphorylated chitosan polyelectrolyte complex. J. Bioact. Compat. Polym. 23, 520-531 (2008).

14 Win, P. P., Shin-ya, Y., Hong, K. J. \& Kajiuchi, T. Formulation and characterization of ph sensitive drug carrier based on phosphorylated chitosan (PCS). Carbohydr. Polym. 53, 305-310 (2003).

15 Sakaguchi, T., Horikoshi, T. \& Nakajima, A. Adsorption of uranium by chitin phosphate and chitosan phosphate. Agric. Biol. Chem. 45, 2191-2195 (1981).

16 Jayakumar, R., Nagahama, H., Furuike, T. \& Tamura, H. Synthesis of phosphorylated chitosan by novel method and its characterization. Int. J. Biol. Macromol. 42, 335-339 (2008).

17 Jayakumar, R., Egawa, T., Furuike, T., Nair, S. V. \& Tamura, H. Synthesis, characterization, and thermal properties of phosphorylated chitin for biomedical applications. Polym. Eng. Sci. 49, 844-849 (2009).

18 Amaral, I. F., Granja, P. L. \& Barbosa, M. A. chemical modification of chitosan by phosphorylation: an XPS, FT-IR and SEM Study. J. Biomater. Sci., Polym. Ed. 16, 1575-1593 (2005).

19 Nishi, N., Nishimura, S., Ebina, A., Tsutsumi, A. \& Tokura, S. Preparation and characterization of water-soluble chitin phosphate. Int. J. Biol. Macromol. 6, 53-54 (1984) 
20 Nishi, N., Ebina, A., Nishimura, S., Tsutsumi, A., Hasegawa, O. \& Tokura, S. Highly phosphorylated derivatives of chitin, partially deacetylated chitin and chitosan as new functional polymers: preparation and characterization. Int. J. Biol. Macromol. 8, 311-317 (1986).

21 Nishi, N., Maekita, Y., Nishimura, S., Hasegawa, O. \& Tokura, S. Highly phosphorylated derivatives of chitin, partially deacetylated chitin and chitosan as new functional polymers: metal binding property of the insolubilized materials. Int. J. Biol. Macromol. 9, 109-114 (1987).
22 Jayakumar, R., Selvamurugan, N., Nair, S. V., Tokura, S. \& Tamura, H. Preparative methods of phosphorylated chitin and chitosan-an overview. Int. J. Biol. Macromol. 43, 221-225 (2008).

23 Whistler, R. L. \& Towle, G. A. Preparation and characterization of polysaccharide phosphates. Arch. Biochem. Biophys. 135, 396-401 (1969).

24 Velde, K. V. \& Kiekens, P. Structure analysis and degree of substitution of chitin, chitosan and dibutyrylchitin by FT-IR spectroscopy and solid state ${ }^{13} \mathrm{C}$ NMR. Carbohydr. Polym. 58, 409-416 (2004). 